\title{
Application of Linear Model Predictive Control and Input-Output Linearization to Constrained Control of 3D Cable Robots
}

\author{
Ali Ghasemi \\ Member of Young Researchers Club, Science and Research Branch, Islamic Azad University, Tehran, Iran \\ E-mail: ali.ghasemi.g@gmail.com \\ Received September 3, 2011; revised October 13, 2011; accepted October 25, 2011
}

\begin{abstract}
Cable robots are structurally the same as parallel robots but with the basic difference that cables can only pull the platform and cannot push it. This feature makes control of cable robots a lot more challenging compared to parallel robots. This paper introduces a controller for cable robots under force constraint. The controller is based on input-output linearization and linear model predictive control. Performance of input-output linearizing (IOL) controllers suffers due to constraints on input and output variables. This problem is successfully tackled by augmenting IOL controllers with linear model predictive controller (LMPC). The effecttiveness of the proposed method is illustrated by numerical simulation.
\end{abstract}

Keywords: Cable Robots, Input-Output Linearization, Linear Model Predictive Control

\section{Introduction}

After a motion simulator with parallel kinematic chains was introduced in 1965 by D. Stewart [1], parallel manipulators received more and more attention because of their high stiffness, high speed, high accuracy, compact and high carrying capability [2]. They have been used widely in the fields of motion simulators, force/torque sensors, compliance devices, medical devices and machine tools $[3,4]$.

A parallel robot is made up of an end-effector, with $n$ degrees of freedom, and a fixed base linked together by at least two independent kinematic chains [5]. Actuation takes place through $\mathrm{m}$ simple actuators. Parallel robots drawbacks are their relatively small workspace and kinematics complexity.

Cable robots are a class of parallel robots in which the links are replaced by cables. They are relatively simple in form, with multiple cables attached to a mobile platform or an end-effector. Cable robots posses a number of desirable characteristics, including: 1) stationary heavy components and few moving parts, resulting in low inertial properties and high payload-to-weight ratios; 2) incomparable motion range, much higher than that obtained by conventional serial or parallel robots; 3 ) cables have negligible inertia and are suitable for high acceleration applications; 4) transportability and ease of disassembly/reassembly; 5) reconfigurability by simply relocating the motors and updating the control system accordingly; and, 6 ) economical construction and maintenance due to few moving parts and relatively simple components $[6,7]$. Consequently, cable robots are exceptionally well suited for many applications such as handling of heavy materials, inspection and repair in shipyards and airplane hangars, high-speed manipulation, rapidly deployable rescue robots, cleanup of disaster areas, and access to remote locations and interaction with hazardous environments [6-12]. For these applications conventional serial or parallel robots are impractical due to their limited workspace.

However, cables have the unique property-they cannot provide compression force on an end-effector. Some research has been previously conducted to guarantee positive tension in the cables while the end-effector is moving. The idea of redundancy was utilized in cable system control $[13,14]$.

This paper introduces a controller for cable robots under force constraint. By considering, linear model predictive covers different constraint such as input constraints. The goal is to apply the linear model predictive control to the input-output linearized system to account for the constraints.

A variety of nonlinear control design strategy has been proposed in the past two decades. Input-output linearization (IOL) and nonlinear model based control are the most widely studied design techniques in nonlinear control. The 
central idea of the input-output linearization approach is to algebraically transform the nonlinear system into linear one and apply a suitable linear control design technique $[15,16]$.

LMPC is primarily developed for process control. Therefore its application in robot control has less been reported. The incipient interest in the applications of MPC dates back to the late 1970s. In 1978, Richalet et al. [17], presented the Model Predictive Heuristic Control (MPHC) method in which an impulse response model was used to predict the effect at the output of the future control actions. Linear model predictive control refers to a class of control algorithms that compute a manipulated variable profile by utilizing a linear process model to optimize a linear or quadratic open-loop performance objective subject to linear constraints over a future time horizon. The first move of this open loop optimal manipulated variable profile is then implemented. This procedure is repeated at each control interval with the process measurements used to update the optimization problem. During 1980s, MPC quickly became popular particularly in chemical process industry due to the simplicity of the algorithm and to the use of the impulse or step response model, which is preferred, as being more intuitive and requiring no previous information for its identification [18].

A cable-suspended robot is actuated by servo motors that control the tensions in the cables. A major disadvantage of cable robots is that each cable can only exert tension. This constraint leads to performance deterioration and even instability, if not properly accounted for in the control design procedure. Due to this feature, well known results in robotics for trajectory planning and control are not directly applicable to them. Several approaches including a lyapunov based controller with variable gains and a feedback linearizing controller with variable gains [19], feedback linearization together with method of reference signal management [20], lyapunov based sliding controller with method of signal management [21] have been suggested to satisfy the positive tension in the cables while the platform is moving.

In this paper a linear model predictive control is applied to linearized model. Model predictive control, a computer control algorithm that utilizes an explicit model to predict the future response of a system is an effective tool for handling constrained control problems.

\section{Kinematics Modeling of the Cable Robots}

The kinematic notation of a spatial cable-driven manipulator is presented in Figure 1, where $P_{i}$ and $B_{i}$ are two attaching points of the $i$ th cable to the platform and the base, respectively. $a_{i}$ represents the position vector of $B_{i}$ in the base frame and bi shows the position vector of the cable connection in the platform frame. Therefore, $T_{i}=$ $a i-R b i-c$ is the vector representing the length of each cable and $l_{i}$ is the direction of tension force along each cable, where $\mathrm{c}$ is the position vector of mass center of platform parameterized. $R$ is the rotation matrix between the two frames, the base and the moving, Figure 1.

\section{System Dynamics}

The inertia of each link of cable robots is negligible compared to that of the platform because the so-called link is just a cable or wire. Therefore, the dynamics of the links can be ignored which will significantly simplify the dynamic model of the manipulator. One can derive the Newton-Euler equations of motion of the manipulator with respect to the center of mass on the platform as follows [10]

$$
\begin{aligned}
& \sum F=m a \Rightarrow m \ddot{c}=f_{\text {ext }}+m g+\sum_{i=1}^{n} T_{i} \\
& (n \text { is number of cables }) \\
& \sum M=I \alpha+\omega \times I \omega \tau \Rightarrow \\
& \tau_{\text {ext }}+\sum_{i=1}^{n}\left(R b_{i}\right) \times\left(f_{i} l_{i}\right)=I \alpha+\omega \times I \omega
\end{aligned}
$$

where $m$ and $I$ are the mass and inertia tensor of the platform including any attached payload; $g$ is the gravity acceleration vector; and $f_{\text {ext }}$ and $\tau_{\text {ext }}$ are external force and moment vectors applied to the platform. $\ddot{c}$ and $\alpha$ are the linear and angular acceleration vectors of the platform; $T_{i}$ and $f_{i}$ are the force vector and force value of the ith cable. Equation (1) can be rewritten into a compact form as:

$$
M \ddot{x}+C \dot{x}+D=J u
$$

where

$$
\begin{aligned}
& \boldsymbol{M}=\left[\begin{array}{cc}
m I_{3 \times 3} & 0_{3 \times 3} \\
0_{3 \times 3} & I
\end{array}\right], \boldsymbol{C}=\left[\begin{array}{cc}
0_{3 \times 3} & 0_{3 \times 3} \\
0_{3 \times 3} & -[I \omega]
\end{array}\right], \\
& \boldsymbol{D}=\left[\begin{array}{c}
f_{\text {ext }}+m \boldsymbol{g} \\
\tau_{\text {ext }}
\end{array}\right]
\end{aligned}
$$

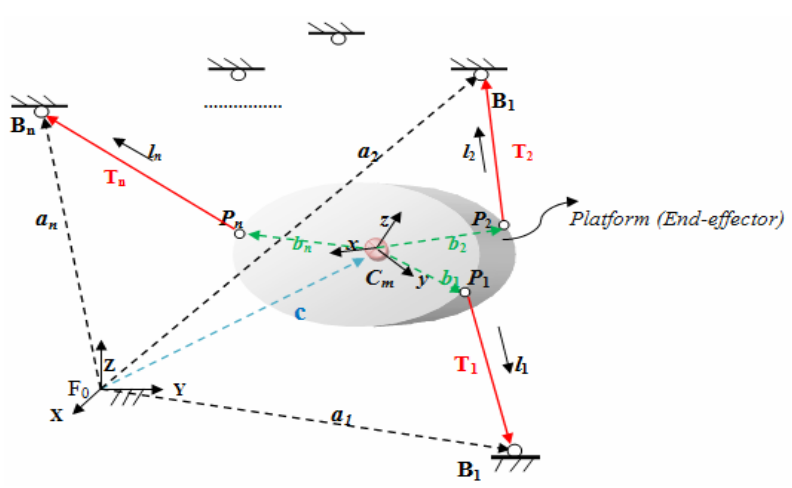

Figure 1. General kinematics of a cable robot. 


$$
\begin{gathered}
\boldsymbol{J}=\left[\begin{array}{c}
l_{1} \cdots l_{n} \\
R b_{1} \times l_{1} \cdots R b_{n} \times l_{n}
\end{array}\right], \boldsymbol{J}=- \text { jacopian matrix }{ }^{T} \\
u=\left[\begin{array}{c}
f_{1} \\
\vdots \\
f_{n}
\end{array}\right]
\end{gathered}
$$

$I_{3 * 3}$ is a $3 * 3$ identity matrix and

$$
[I \omega]=\left[\begin{array}{ccc}
0 & -(I \omega)_{z} & (I \omega)_{y} \\
(I \omega)_{z} & 0 & -(I \omega)_{x} \\
-(I \omega)_{y} & (I \omega)_{x} & 0
\end{array}\right]
$$

Equation (2) can be written into a steady state form as:

$$
\begin{aligned}
& \dot{X}=F+G u \\
& y=E X=h(X)
\end{aligned}
$$

where

$$
\begin{aligned}
& y=I x \\
& X=\left\{\begin{array}{l}
x \\
\dot{x}
\end{array}\right\}, F=\left\{\begin{array}{c}
\dot{x} \\
-M^{-1}(C \dot{x}+D)
\end{array}\right\}, G=\left\{\begin{array}{c}
0_{6^{*} n} \\
M^{-1} J
\end{array}\right\}
\end{aligned}
$$

with constraints

$$
0 \leq u \leq u_{\max }
$$

\section{Input-Output Linearization}

The difficulty of the tracking control design can be reduced if we can find a direct and simple relation between the system output $y$ and the control input $u$. Indeed, this idea constitutes the intuitive basis for the so-called input -output linearization approach to nonlinear control design.

This article is aimed to use the LMPC which covers different constraints such as input constraints. Because of LMPC is usually used for linear discrete systems. In the beginning we will linearize the dynamics equations based on in Input-Output Linearization.

To generate a direct relationship between the output $y$ and the input $u$, Differentiate the output $y_{i}$ with respect to time $t$ in Equation (3), we have

$$
y_{i}=L_{F} h_{i}(X)+\sum_{j=1}^{n} L_{g_{j}} h_{i} u_{j}(t)
$$

where $L_{F} h_{i}(X)$ and $L_{g j} h_{i}(X)$ are the Lie derivatives of $h_{i}(X)$ with respect to $F(X)$ and $g_{j}(X)$, respectively. If $L_{g j} h_{i}(\mathrm{X})=0, y_{i}=L_{F} h_{i}(X)$ then the $r_{i}$ th derivative of $y_{i}$ can be represent in the following form.

$$
y_{i}^{r_{i}}=L_{F}^{r_{i}} h_{i}(X)+\sum_{j=1}^{n} L_{g_{j}} L_{F}^{r_{i}-1} h_{i} u_{j}(t)
$$

In this way, we can write all plant's input-output equations as

$$
\left[\begin{array}{c}
y_{1}^{r_{1}} \\
\vdots \\
y_{q}^{r_{q}}
\end{array}\right]=\left[\begin{array}{c}
L_{F}^{r_{1}} h_{1} \\
\vdots \\
L_{F}^{r_{q}} h_{q}
\end{array}\right]+\left[\begin{array}{c}
L_{g_{1}} L_{F}^{r_{1}-1} h_{1} \cdots L_{g_{n}} L_{F}^{r_{1}-1} h_{1} \\
\\
L_{g_{1}} L_{F}^{r_{q}-1} h_{q} \cdots L_{g_{n}} L_{F}^{r_{q}-1} h_{q}
\end{array}\right] u
$$

where $q$ and $r_{i}$ are the number of degree of freedom of the robot and the relative order of the plant, respectively.

Equation (4) can be represented in the following compact form:

$$
v=P+W u
$$

Now u can be obtained as:

$$
u=\left(W^{*} W\right)^{-1} W^{*}(v-P)
$$

where $W^{*}$ is the pseudo inverse of $W$.

$$
W^{*}=W^{T}\left(W W^{T}\right)^{-1}
$$

For a cable robotic system, it can be easily shown that the system has no zero dynamics. Therefore, the decoupling matrix $\mathrm{W}$ is full rank and the control law is well defined and a suitable change of coordinates $\xi^{T}=\Phi(X)$ where $\Phi(X)=\left[\begin{array}{lllllll}y_{1} & \dot{y}_{1} & y_{2} & \dot{y}_{2} & \cdots & y_{q} & \dot{y}_{q}\end{array}\right]^{T}$ yields a closedloop system in the normal form [15].

$$
\begin{gathered}
y=x \Rightarrow r_{i}=2 \quad i=1, \cdots, q \\
\Rightarrow r_{1}+\cdots+r_{q}=n \\
\dot{\xi}=A \xi+B v \\
y=H \xi
\end{gathered}
$$

\section{Linear Model Predictive Control}

The basic structure of MPC to implement is shown in Figure $2 \mathrm{~A}$ model is used to predict the future plant outputs, based on past and current values and on proposed future control actions. These actions are calculated by optimizer taking into account the cost function as well as constraints. The optimizer is another fundamental part of the strategy as it provides the control action. each component of this structure is described in more detail In the following of this article.

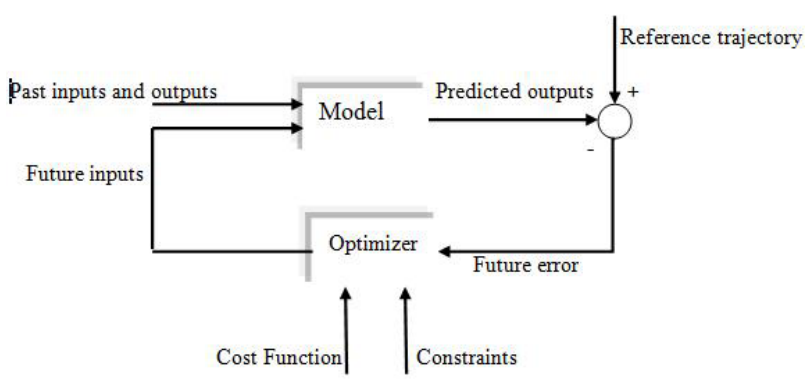

Figure 2. Basic structure of MPC. 
The goal is to apply the linear model predictive control to the input-output linearized system to account for the constraints. Since the linear model predictive is more naturally formulated in discrete time, the linear subsystem in (5) is discretized with a sampling period $T$ to yield

$$
\begin{aligned}
& \xi(k+1)=A_{d} \xi(k)+B_{d} v(k) \\
& y(k)=H_{d} \xi(k)
\end{aligned}
$$

where $A_{d}, B_{d}$ and $H_{d}$ are obtained directly from the continuous-time matrices [22].

Also, the state-dependent relation between $u(k)$ and $v(k)$ is obtained as

$$
v(k)=L_{F}^{r} h[X(k)]+L_{G} L_{F}^{r} h[X(k)] u(k)
$$

This mapping can be rewritten in the following form

$$
v(k)=P[X(k)]+W[X(k)] u(k)
$$

\subsection{Constraint Mapping}

When linear model predictive control is applied to the system, it is necessary to map the constraints from the original input space to linearized system. By considering $v$ is a new input to be determined, To obtain constraints on the new input, The input constraint mapping is performed using input-output linearization law and the current state measurement $x(k)$. The transformed constraints can be determined on each sampling period by solving the following optimization problem:

$$
\begin{aligned}
v_{\min }(k+j \mid k)= & \min _{u(k+j \mid k)} P[X(k+j \mid k)] \\
& +W[X(k+j \mid k)] u(k+j \mid k) 0 \leq j \leq N-1 \\
v_{\max }(k+j \mid k)= & \max _{u(k+j \mid k)} P[X(k+j \mid k)] \\
& +W[X(k+j \mid k)] u(k+j \mid k) 0 \leq j \leq N-1 \\
u_{\min } \leq u(k+j \mid j) \leq & u_{\max }
\end{aligned}
$$

where $Z(k+j \mid k)$ is the predicted value of the system state $(Z)$ at time $\mathrm{k}+\mathrm{j}$ based on the information available at time $k$.

Note that the variable $X(k+j \mid k)$ cannot be calculated provided that the input sequence is calculated, which is not possible until the constraints are specified. Therefore, at the beginning $(k=0)$, the input constraint over the entire control horizon can be presented by:

$$
\begin{aligned}
& v_{\text {min }}(k+j \mid k)=v_{\text {min }}(k \mid k) \\
& v_{\text {max }}(k+j \mid k, N-1 \\
& j=1, \cdots, N-1
\end{aligned}
$$

Then, we use inputs calculated at last sampling time to determine future constraints at the current sampling time.
Therefore, Equation (7) will be changed into Equation (9).

$$
\begin{aligned}
& v_{\min }(k+j \mid k)= \min _{u(k+j \mid k)} P[X(k+j \mid k-1)] \\
&+W[X(k+j \mid k-1)]] u(k+j \mid k) 0 \leq j \leq N-1 \\
& v_{\max }(k+j \mid k)= \max _{u(k+j k)} P[X(k+j \mid k-1)] \\
&+W[X(k+j \mid k-1)] u(k+j \mid k) 0 \leq j \leq N-1 \\
& u_{\min } \leq u(k+j \mid j) \leq u_{\max }
\end{aligned}
$$

Now Equation (9) can be solved to obtain $v_{\min }(k+j \mid k)$ and $v_{\max }(k+j \mid k)$. If $W(i, j)$ is positive, the control $u(j)$ must be the smallest value for $v_{\min }$ and the largest value for $v_{\max }$ and if $W(i, j)$ is negative, then it must be the largest value for $v_{\min }$ and the smallest value for $v_{\max }$.

\subsection{Linear Model Predictive Control Design}

The goal is to apply LMPC to the linearized system to account for these constraints. Now the model (6) is used in the infinite horizon linear model predictive strategy proposed by Muske and Rawlings [23]. Therefore, the openloop optimal control problem that the input control found by minimizing the infinite horizon criterion, can be expressed as

$$
\begin{aligned}
& \min _{V(k \mid k)} \sum_{j=1}^{\infty}\left(\xi(k+j \mid k)-\xi_{\mathrm{d}}\right)^{T} H_{\mathrm{d}}^{T} Q H_{\mathrm{d}}\left(\xi(k+j \mid k)-\xi_{\mathrm{d}}\right) \\
& +\left(v(k+j \mid k)-v_{\mathrm{d}}\right)^{T} S\left(v(k+j \mid k)-v_{\mathrm{d}}\right) \\
& +(v(k+j \mid k)-v(k+j-1 \mid k))^{T} \\
& \times R(v(k+j \mid k)-v(k+j-1 \mid k))
\end{aligned}
$$

where $\xi_{d}$ and $v_{d}$ are target values for $\xi$ and $v$, respectively, and $Q, S$, and $R$, are positive semi definite matrices.

In order to obtain value $v(k+j \mid k)$ it is necessary to minimize the functional of Equation (10) to do this value of the predicted output are calculated as function of pas values of inputs and outputs and future control signals obtain an expression whose minimization leads to the looked for values.

The decision vector is defined as $V(k \mid k)=[v(k \mid k)$ $v(k+1 \mid k) \cdots v(k+N-1 \mid k)]^{T}$, where $N$ is the control horizon. All future moves beyond the control horizon are set equal to the target value $v_{d}$. As discussed in [23], the matrix $A_{d}$ is unstable and in order for the linear model predictive problem to have a feasible solution it is necessary to impose the equality constraint $\xi(k+N \mid k)=\xi_{d}$. To obtain a finite set of decision variables, inputs beyond the control horizon are set equal to the desired value: $v(k+$ $j \mid k)=v_{d}, j \geq N$. Therefore, the infinite horizon linear 
model predictive problem Equation (10) can be written as a finite horizon problem.

$$
\begin{aligned}
& \min _{V(k \mid k)}\left(v(k+N-1 \mid k)-v_{\mathrm{d}}\right)^{T} S\left(v(k+N-1 \mid k)-v_{\mathrm{d}}\right) \\
& +\sum_{j=1}^{N-1}\left(\left(\xi(k+j \mid k)-\xi_{\mathrm{d}}\right)^{T} H_{\mathrm{d}}^{T} Q H_{\mathrm{d}}\left(\xi(k+j \mid k)-\xi_{\mathrm{d}}\right)\right. \\
& +\left(v(k+j \mid k)-v_{\mathrm{d}}\right)^{T} S\left(v(k+j \mid k)-v_{\mathrm{d}}\right) \\
& +(v(k+j \mid k)-v(k+j-1 \mid k))^{T} \\
& R(v(k+j \mid k)-v(k+j-1 \mid k)))
\end{aligned}
$$

This optimization problem must be solved subjected to the following constraints:

$$
\begin{aligned}
& \xi(k+N \mid k)=\xi_{d} \\
& v_{\text {min }}(k+j \mid k) \leq v(k+j \mid k) \leq v_{\min }(k+j \mid k)
\end{aligned}
$$

Straightforward algebraic manipulation of quadratic objective function of the corresponding regular system presented in Equation (11) results in the following standard quadratic program form:

$$
\min _{V(k \mid k)} V^{T}(H H) V+2 V^{T}[(G G) \xi(k)-(F F) v(k-1)]
$$

where

$$
\boldsymbol{G G}=\left[\begin{array}{c}
B_{d}^{T} K_{N-1} A_{d} \\
\vdots \\
B_{d}^{T} K_{0} A_{d}
\end{array}\right], F F=\left[\begin{array}{c}
S \\
0 \\
\vdots \\
0
\end{array}\right]
$$

Equation (13) should be solved subjected to the following constraints:

$$
\begin{aligned}
& (D D) V \leq(C C) \\
& (E E) V=A_{d}^{N} \xi(k)
\end{aligned}
$$

where by considering

$$
\mathbf{D} \boldsymbol{D}=\left[\begin{array}{c}
I_{6^{*} N} \\
-I_{6^{*} N}
\end{array}\right], \boldsymbol{C} \boldsymbol{C}=\left[\begin{array}{c}
v_{\max }(k \mid k) \\
\vdots \\
v_{\max }(k+N-1 \mid k) \\
-v_{\min }(k \mid k) \\
\vdots \\
-v_{\min }(k+N-1 \mid k)
\end{array}\right],
$$

$$
\begin{aligned}
& \boldsymbol{E} \boldsymbol{E}=\left[\begin{array}{llll}
A_{d}^{N-1} & B_{d} & A_{d}^{N-2} & B_{d} \\
\cdots & B_{d}
\end{array}\right] \\
& K_{N}=\sum_{i=0}^{N} A_{\mathrm{d}}^{T^{i}}\left(H_{\mathrm{d}}^{T} Q H_{\mathrm{d}}\right) A_{\mathrm{d}}^{i}
\end{aligned}
$$

The solution of Equation (13) belongs to the regular system. To find the solutions of the tracking one, that of the regular system should be shifted into the origin of the system to the steady state described by $\xi_{d}$, and $v_{d}$. The desired values must lie within the feasible region defined by input constraints for linear model predictive control and minimize the control effort (Equation (15)).

$$
\begin{gathered}
\min _{U_{d}}\left(\left(\left[\begin{array}{ll}
0_{m \times 2 m} & I_{m \times m}
\end{array}\right]\left(U_{d}-\bar{U}\right)\right)^{T} \times Q_{s} \times\right. \\
\left(\left[\begin{array}{ll}
0_{m \times 2 m} & I_{m \times m}
\end{array}\right]\left(U_{d}-\bar{U}\right)\right)^{T} \\
U_{d}=\left[\begin{array}{ll}
\xi_{d} & v_{d}
\end{array}\right] \\
\text { subject } \text { to : } \\
{\left[\begin{array}{cc}
I-A_{d} & B_{d} \\
H_{d} & 0
\end{array}\right] U_{d}=\left[\begin{array}{c}
0 \\
y_{d}
\end{array}\right]} \\
{\left[\begin{array}{ll}
0_{2 m^{*} 2 m} & D D
\end{array}\right] U_{d} \leq C}
\end{gathered}
$$

$\bar{U}$ and Qs are desired value of the input and a positive definite matrix, respectively. Therefore control law is the summation of the answers of Equation (13) and Equation (15) which can be shown in the following form.

$$
\begin{aligned}
& u(k)=W^{*}(X(k \mid k))(v(k \mid k)-P(X(k \mid k)) \\
& +W^{*}\left(X_{d}(k \mid k)\right)\left(v_{d}(k \mid k)-P\left(X_{d}(k \mid k)\right)\right.
\end{aligned}
$$

Figure 3 shows schematic of the proposed structure control.

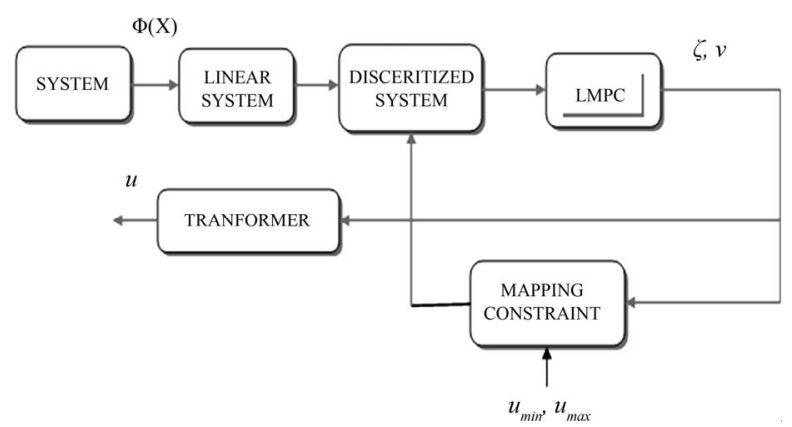

Figure 3. Schematic of control structure.

$$
\boldsymbol{H H}=\left[\begin{array}{cc}
B_{d}^{T} K_{N-1} B_{d}+R+2 S & B_{d}^{T} A_{d}^{T} K_{N-2} B_{d}-S \cdots B_{d}^{T} A_{d}^{T^{N-1}} K_{0} B_{d} \\
B_{d}^{T} K_{N-2} A_{d} B_{d}-S & B_{d}^{T} K_{N-2} B_{d}+R+2 S \cdots B_{d}^{T} A_{d}^{T^{N-2}} K_{0} B_{d} \\
\vdots & \vdots \\
B_{d}^{T} K_{0} A_{d}^{N-1} B_{d} & B_{d}^{T} K_{0} A_{d}^{N-2} B_{d} \cdots B_{d}^{T} K_{0} B_{d}+R+2 S
\end{array}\right]
$$




\section{Simulation}

In this section, simulation results of applying model predictive control on a 3D cable robot will be presented. Table 1. shows the dimensions of the cable robot. We consider a definite movement of the platform from $\mathrm{X}_{0}=$ $[-0.1,-0.1,1.5,0.002, .001,-0.001]^{\mathrm{T}}$ to $\mathrm{X}=[0.2 \sin (\mathrm{t}), 0.4$ $\left.\sin (\mathrm{t}), 1+0.2 \mathrm{t}^{2}-0.03 \mathrm{t}^{3}, 0,0,0\right]^{\mathrm{T}}$, on a desired trajectory shown in dotted lines by Figures 4(a) to 4(f), for position/orientation of the platform.

Also, we consider the parameters of the model predicttive controller as: the control horizon $N=25, S, Q$, and $R$ of Equaqion (10), $R=I, Q=I, S=0.011$, and $Q_{s}$ of Equation (15), $Q_{s}=I$.

Since the controller needs platform's position/orienttation, at first, we must solve forward kinematics of the robot. This has been carried out by the authors using neural network algorithm [24].

Figure 4 shows the model predictive controller with input-out linearizing worked quite well and a trajectory tracking are done. Figure 5 shows the six tensions in the cables vs. time. As it can be seen, all of them remain positive during the motion.

Table 1. Dimensions of the cable robot.

\begin{tabular}{cccccccc}
\hline Position vector & $\mathrm{X}(\mathrm{m})$ & $\mathrm{Y}(\mathrm{m})$ & $\mathrm{Z}(\mathrm{m})$ & Position vector & $\mathrm{x}(\mathrm{m})$ & $\mathrm{y}(\mathrm{m})$ & $\mathrm{z}(\mathrm{m})$ \\
\hline $\mathrm{a}_{1}$ & 1.1547 & -2 & 3 & $\mathrm{~b}_{1}$ & -0.2887 & -0.5 & 0 \\
$\mathrm{a}_{2}$ & 1.1547 & -2 & 3 & $\mathrm{~b}_{2}$ & 0.5774 & 0 & 0 \\
$\mathrm{a}_{3}$ & 1.1547 & 2 & 3 & $\mathrm{~b}_{3}$ & 0.5774 & 0 & 0 \\
$\mathrm{a}_{4}$ & 1.1547 & 2 & 3 & $\mathrm{~b}_{4}$ & -0.2887 & 0.5 & 0 \\
$\mathrm{a}_{5}$ & -2.309 & 0 & 3 & $\mathrm{~b}_{5}$ & -0.2887 & 0.5 & 0 \\
$\mathrm{a}_{6}$ & -2.309 & 0 & 3 & $\mathrm{~b}_{6}$ & -0.2887 & -0.5 & 0
\end{tabular}

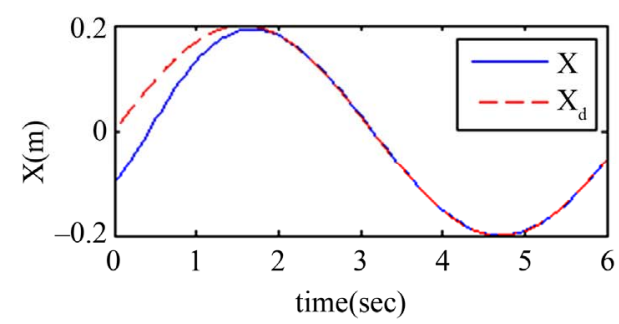

(a)

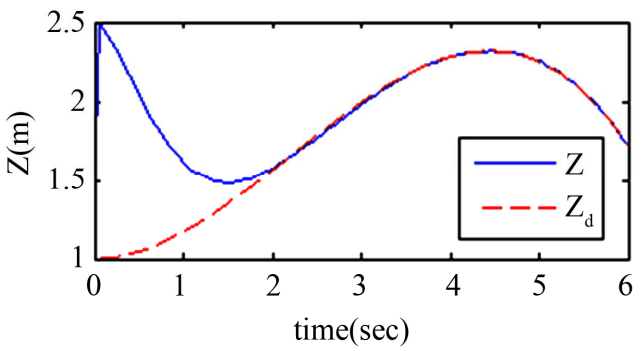

(c)

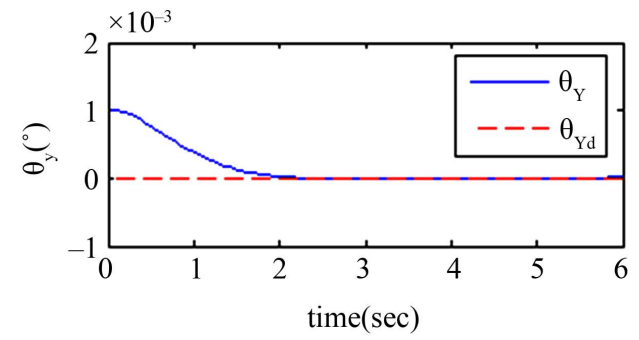

(e)

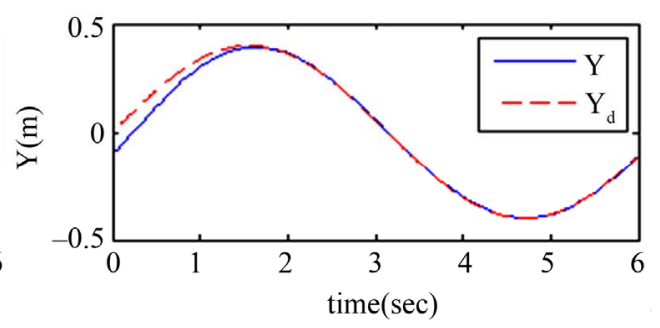

(b)

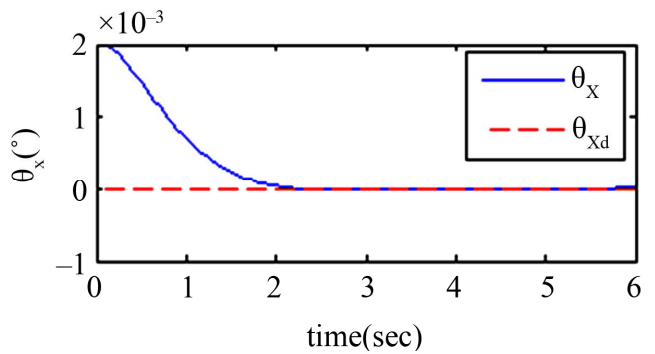

(d)

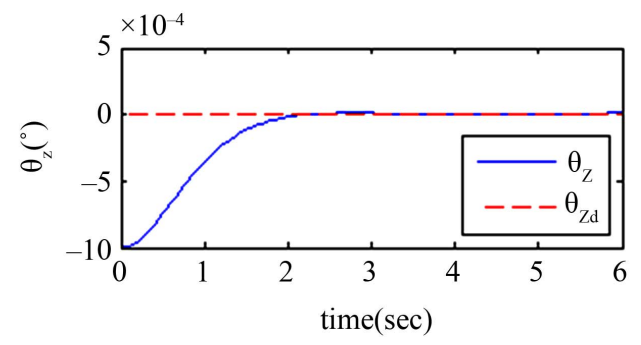

(f)

Figure 4. Plots of desired and actual position and orientation of the platform, (a)-(c) position in X-Y-Z directions, respectively, (d)-(f) orientation around $\mathrm{X}-\mathrm{Y}-\mathrm{Z}$ direction, respectively. 


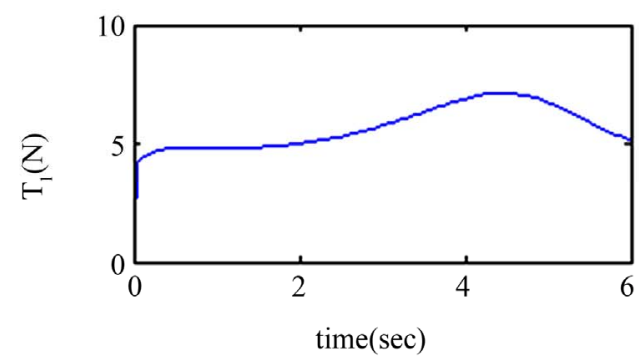

(a)

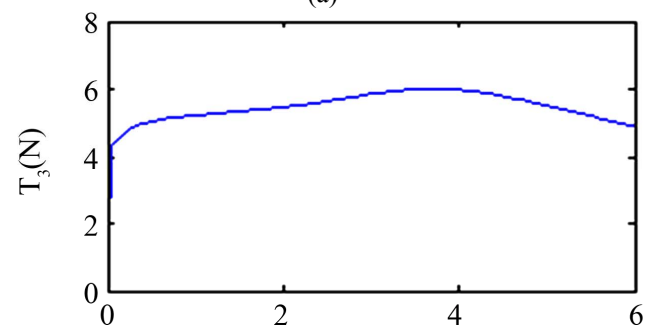

(c)

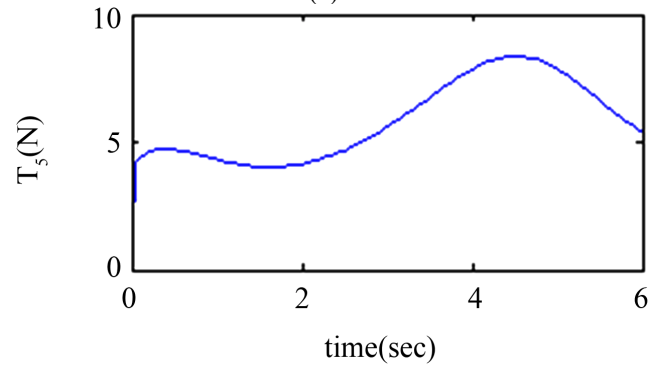

(e)

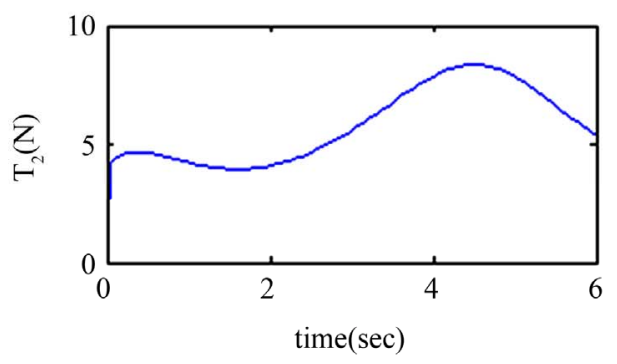

(b)

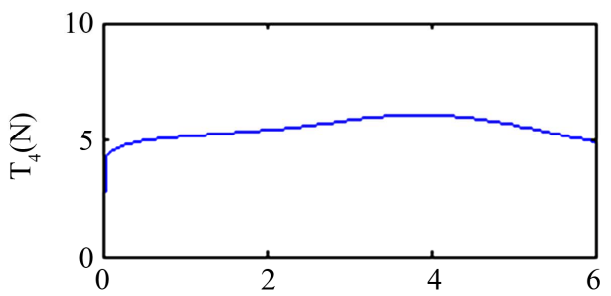

(d)

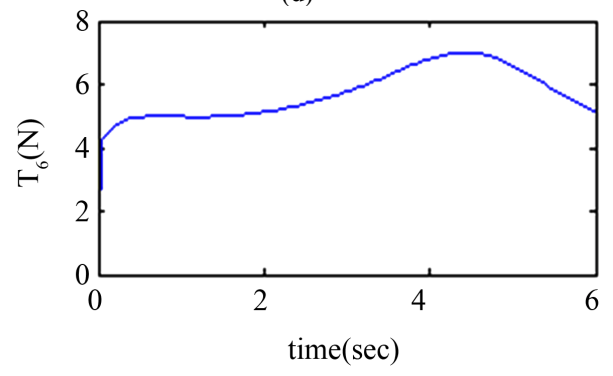

(f)

Figure 5. Plots of tension trajectories.

\section{Conclusions}

In this paper, a linear model predictive controller together with an input-output linearizing control strategy for a constrained robotic system, a 3D cable robot, was developed and evaluated. The control system is comprised of: 1) an input-output linearizing controller that accounts for cable robot nonlinearities; 2) a constraint mapping scheme that transforms the actual input constraints into input constraints on the feedback linearized system; and 3) a linear model predictive controller that provides explicit compensation for input constraints. The simulation results showed the effectiveness of the proposed method. It is worth nothing that this approach can be extended for the redundant cable robots.

\section{References}

[1] D. Stewart, "A Platform with Six Degrees of Freedom," Proceedings of the Institution of Mechanical Engineer, Vol. 180, No. 15, 1965, pp. 371-386. doi:10.1243/PIME_PROC_1965 $180 \_02902$

[2] J. -P. Merlet, "Parallel Robots, Solid Mechanics and Its
Applications," Kluwer, Norwell, 2001.

[3] J. -P. Merlet, "Still a Long Way to Go on the Road for Parallel Mechanisms," A Keynote Speech at Design Engineering Technical Conferences, Montreal, 29 September-2 October 2002.

[4] J. -P. Merlet, "Parallel Robots, Open Problems," INRIA Sophia-Antipolis, France. http://www.-sop.inria.fr

[5] L. -W. Tsai, "Robot Analysis, The Mechanics of Serial and Parallel Manipulators," Wiley, New York, 1999.

[6] P. Bosscher, A. T. Riechel and I. Ebert-Uphoff, "WrenchFeasible Workspace Generation for Cabledriven Robots," Journal of Intelligent and Robotic Systems, Vol. 22, No. 4, 2006, pp. 890-902.

[7] A. T. Riechel and I. Ebert-Uphoff, "Force-Feasible Workspace Analysis for Underconstrained Pointmass Cable Robots," Proceedings of IEEE International Conference on Robotics and Automation, New Orleans, 26 April-1 May 2004, pp. 4956-4962.

[8] S. Kawamura, W. Choe, S. Tanaka and S. R. Pandian, "Development of an Ultrahigh Speed FALCON Using Wire Drive System," In Proceedings of the 1995 IEEE International Conference on Robotics and Automation, 21-27 May 2003, pp. 215-220.

[9] P. Lafourcade, M. Llibre and C. Reboulet, "Design of a Par- 
allel Wire-Driven Manipulator for Wind Tunnels," In Proceedings of the Workshop on Fundamental Issues and Future Research Directions for Parallel Mechanisms and Manipulators, Quebec, 3-4 October 2002, pp. 187-194.

[10] X. Diao, O. Ma and R. Paz, "Study of 6-DOF Cable Robots for Potential Application of HIL Microgravity Contact-Dynamics Simulation," In Proceedings of the AIAA Modeling and Simulation Technologies Conference and Exhibit, Keystone, 21-24 August 2006, pp. 1097-1110.

[11] P. Gallina, G. Rosati and A. Rossi, "3-D.O.F. Wire Driven Planar Haptic Interface," Journal of Intelligent and Robotic Systems, Vol. 32, No. 1, 2001, pp. 23-36. doi:10.1023/A:1012095609866

[12] J. Albus, R. Bostelman and N. Dagalakis, "The NIST Robocrane," Journal of Robotic Systems, Vol. 10, No. 5, 1993, pp. 709-724. doi:10.1002/rob.4620100509

[13] Y. Q. Zheng, "Workspace Analysis of a Six DOF WireDriven Parallel Manipulator," Proceedings of the WORKSHOP on Fundamental Issues and Future Research Derection for Parallel Mechanisms and Manipulators, Quebec, 3-4 October 2002, pp. 287-293.

[14] W. J. Shiang, D. Cannon and J. Gorman, "Dynamic Analysis of the Cable Array Robotic Crane," Proceedings of the IEEE International Conference on Robotics and Automation, Detroit, 10-15 May 1999, pp. 2495-2500.

[15] J. J. Slotine and W. Leiping, "Applied Nonlinear Control," Prentice Hall, Englewood Cliffs, 1991.

[16] H. Khalil, "Nonlinear Systems," 3rd Edition, Prentice-Hall, Upper Saddle River, 2002.

[17] J. Richalet, A. Raault, J. L. Testud and J. Papon, "Model
Predictive Heuristic Control: A pplication to Industry Processes," Automatica, Vol. 14. No. 2, 1978, pp. 413-428. doi:10.1016/0005-1098(78)90001-8

[18] C. E. Garcia, D. M. Prett and Morari, "Model Predictive Control: Theory and Practice-a Survey," Automatica, Vol. 25, No. 3 1989, pp. 335-348. doi:10.1016/0005-1098(89)90002-2

[19] A. B. Alp and A. K. Agrawal, "Cable Suspended Robots: Design, Planning and Control," International Conference on Robotics Robotics \& Automation, Washington, DC, 913 May 2002, pp. 556-561.

[20] S. R. Oh and A. K. Agrawal, "Controller Design for a Non-redundant Cable Robot Under Input Constraint," ASME International Mechanical Engineering Congress \& Exposition, 16-21 November 2003, Washington, DC.

[21] S. R. Oh and A. K. Agrawal, "A Control Lyapunov Approach for Feedback Control of Cable-Suspended Robots," IEEE International Conference on Robotics and Automation, 10-14 April 2007, pp. 4544-4549.

[22] F. Franklin, J. Powell and L. Workman "Digital Control of Dynamic Systems" 2nd Edition, Addison Wesley, Boston, 1994, pp. 40-70.

[23] K. R. Muske and J. B. Rawlings, "Model predictive control with linear models," AIChE Journal, Vol. 39, No. 2, 1993, pp. 262-287. doi:10.1002/aic.690390208

[24] A. Ghasemi, M. Eghtesad and M. Farid "Neural Network Solution for Forward Kinematics Problem of Cable Robot," Journal of Intelligent and Robotic Systems, Vol. 60, No. 2, 2010, pp. 201-215. doi:10.1007/s10846-010-9421-Z 